\title{
Experimental signatures of spiky local density of states in quasicrystals
}

\author{
Roland Widmer, ${ }^{1, *}$ Pierangelo Gröning, ${ }^{1}$ Michael Feuerbacher ${ }^{2}$ and Oliver Gröning ${ }^{1}$ \\ ${ }^{1}$ EMPA Swiss Federal Laboratories for Materials Testing and Research, nanotech@surfaces, Feuerwerkerstrasse 39, \\ CH-3602 Thun, Switzerland \\ ${ }^{2}$ Institut für Festkörperforschung, Forschungszentrum Jülich, Jülich D-52425, Germany \\ (Received 26 September 2008; revised manuscript received 9 February 2009; published 17 March 2009)
}

\begin{abstract}
Our recent investigations on the valence electronic structure of the icosahedral $\mathrm{Al}_{70} \mathrm{Pd}_{21} \mathrm{Mn}_{9}$ quasicrystals by means of scanning tunneling microscopy and spectroscopy at low temperatures $(5 \mathrm{~K})$ have revealed signatures of a spiky local density of states near the Fermi energy. The local tunneling spectra are characterized by narrow peaks and pseudogaps exhibiting typical energy width of 20-50 meV. The spectroscopic features are of very localized nature, with a typical spatial extent of $0.5-1 \mathrm{~nm}$. This observation reanimates the discussion of the nature of the valence electronic states in aperiodic crystals, where Bloch's theorem-fundamental to the band picture in periodic crystals - cannot be applied. We observe that the spiky aspect of the density of states is only present on the nanometer scale. If series of local point spectra recorded over surface areas larger than typically $10 \times 10 \mathrm{~nm}^{2}$ are averaged, the multitude of local features is smeared out and lead to a rather smooth total spectrum.
\end{abstract}

DOI: 10.1103/PhysRevB.79.104202

PACS number(s): 71.23.Ft, 68.37.Ef, 68.35.B-, 73.20.-r

\section{INTRODUCTION}

Quasicrystals (QCs) are long-range-ordered aperiodic intermetallic alloys possessing fivefold, eightfold, tenfold, or twelvefold rotational symmetries forbidden by classical crystallography. ${ }^{1}$ The absence of translational symmetry, however, in combination with a highly ordered atomic arrangement and rotational symmetry are the characteristics of quasicrystalline systems. QC exhibit very particular mechanic, magnetic, electronic transport, and surface properties, which have been partly associated with the unique aperiodic structural properties of this class of materials situated between crystalline and disordered solids. ${ }^{2}$ As an example, icosahedral QCs composed of $\mathrm{Al}$ and transition metals exhibit an unusually high electric resistivity, considerably higher than the one of the constituting metals, with a negative temperature coefficient. This behavior strongly resembles materials at the Mott-Anderson metal-insulator transition. ${ }^{3}$ Among the different particular properties of QC, the physical characteristics related to the valence electronic structure of the quasiperiodicity are of particular interest, as the Bloch theorem-fundamental to the electron band picture of classical metals-cannot be applied anymore and the concept of a well-defined Brillouin zone and associated zone folding breaks down. On the other hand, strong and sharp Bragg peaks can be observed, which can be used to define pseudo-Brillouin zones with the positions of these strong Bragg peaks as zone centers. ${ }^{4}$

In this context the question arises, whether the quasicrystalline structure leads to the localization of states and whether this localization is at the origin of the exceptional electronic transport properties, with the electrical resistivity decreasing with increasing structural disorder in QC materials. ${ }^{3,5}$ This behavior, unlike the temperature coefficient of the resistivity, is contrary to the one expected for a system showing Anderson localization and may be explained by the situation that static disorder destroys the quasiperiodicity of the system and thereby spikes in the density of states (DOS) are smeared out and channels of transport open. ${ }^{6}$ Recently, a new mechanism for the conductivity in those systems where the velocity of charge carriers is very low has been presented. ${ }^{7}$ This mechanism is related with a specific localization of electrons by complex atomic structures. Even though early theoretical works ${ }^{8}$ predicted a strongly localized valence electron system and-as a consequence-a spiky DOS, more recent theoretical ${ }^{9}$ and also experimental results $^{10-18}$ seem to point to a nonspiky total DOS. However, the existence of spiky DOS in quasicrystals and approximants with large unit cells is still much under debate., $, 19,20$

A general feature of the QC total DOS at the Fermi energy $\left(E_{F}\right)$ is a large Hume-Rothery-type pseudogap in the order of $1 \mathrm{eV}$ width, which has been theoretically predicted and observed by several experimental techniques. ${ }^{19,21}$ Calculations of the DOS on quasiperiodic tilings ${ }^{9,22}$ or large approximants by $a b$ initio methods ${ }^{23,24}$ in the range of the pseudogap predict additionally sets of peaks and gaps with small width in the range of 10-100 meV. Up to now, only little experimental information about this fine structure could be revealed and neither systematic theoretical nor experimental investigations of the local DOS (LDOS) are found in the literature. This, even though it has been shown that the short-range order (typical length $2 \mathrm{~nm}$ ) was the most important parameter to explain the high resistivity and the anomalous order dependency of QC, as the conductivity increases with increasing disorder. ${ }^{2,22,25}$ Difficulties in the experimental investigation of the fine structure in the LDOS arise from the requirement of a high-energy resolution, as the peaks and gaps in question are only a few meV wide. Furthermore, a high spatial resolution is required due to the sensitivity of the features on local order and chemical composition.

These requirements point to investigations using highresolution scanning tunneling microscopy and spectroscopy (STM/STS) as we present in this work. An indispensable prerequisite of this technique is, however, that atomically well-defined clean QC surfaces can be prepared. Up to now, the low-temperature (LT) tunneling spectroscopy on QC has only been performed on oxidized surfaces., ${ }^{3,10}$ Due to the investigation techniques and the oxide layer, the surface structure is not well defined, mostly unknown, and no correlation with the topography could be achieved. In a previous 
paper, ${ }^{20}$ we focused on the occurrence of electronic states and the narrow pseudogap very close to the Fermi energy $E_{F}$ and reported about their spatial distribution on the clean fivefold surface of AlPdMn.

In this paper, our discussion concentrates on the high spatial variability of the LDOS, the correlation with the total DOS, and the development of the narrow pseudogap at the Fermi energy $\left(E_{F}\right)$. This narrow pseudogap is identified as the zero-bias anomaly (ZBA) according to the electronelectron interaction as explained theoretically by Altshuler and Aronov. ${ }^{26}$ To reveal these findings, we accomplished STM and STS investigations on the fivefold and twofold surfaces of icosahedral (i-) $\mathrm{Al}_{70} \mathrm{Pd}_{21} \mathrm{Mn}_{9}$.

\section{EXPERIMENT}

The fivefold and twofold surfaces of a large $(\varnothing 7 \mathrm{~mm})$ Czochralski-grown single grain $i$ - $\mathrm{Al}_{70} \mathrm{Pd}_{21} \mathrm{Mn}_{9} \mathrm{QC}$ were mechanically polished with diamond paste down the $0.1 \mu \mathrm{m}$ grain size. After insertion into vacuum, the crystal was prepared using several sputtering and annealing cycles in order to obtain a bare-terraced $i$-AlPdMn surface. $\mathrm{The}^{+}{ }^{+}$sputtering was carried out at a grazing angle of about $30^{\circ}$ with respect to the surface plane. After annealing the QC at 900 $\mathrm{K}$, the temperature was gently decreased down to $570 \mathrm{~K}$ over a period of about $1 \mathrm{~h}$. Subsequent LEED shows sharp spots from 8 up to $320 \mathrm{eV}$. This procedure is known to produce bare bulk-terminated fivefold ${ }^{27}$ and twofold ${ }^{28}$ QC surfaces.

STM imaging and STS measurements using a lock-in technique were performed with an Omicron LT STM at 5.3 $\mathrm{K}$ and at a base pressure below $5 \times 10^{-11}$ mbar with a mechanically cut Pt/Ir tip. The typical parameters for the lock-in STS measurements were 2.5-10 $\mathrm{mV}$ amplitude of the ac signal and a frequency of $280 \mathrm{~Hz}$. The single $d I / d V$ curves for specific spots on the sample surface are obtained from STS grid measurements, where a large set of equidistant $d I / d V$ spectra is measured during the topography scan. For a more clear identification of the fine structure in the electronic states, the spectra represented in Figs. 5 and 6 are background subtracted by the large parabola originating from the Hume-Rothary pseudogap. This subtraction was applied to every single spectrum with a best-fit parabola and normalized to have the same minimum and maximum in $d I / d V$.

Due to the lack of specific theoretical information ${ }^{22}$ on the local density of states of QC surfaces, we have performed $s$-wave tight-binding (TB) simulations on a finite patch of the Penrose tiling, which can serve as model structures of aperiodic surfaces. The vertices of the Penrose tiling have been taken as grid points for the definition of the Hamiltonian matrix with a nondirectional hopping integral of the type $\mathbf{H}_{i i}=0$ and $\mathbf{H}_{i j}=-\varepsilon_{0} \exp \left(-\mathbf{c}\left|\mathbf{r}_{i}-\mathbf{r}_{j}\right|\right)$. The local density of states at a given vertex point $\mathbf{r}_{j}$ has been calculated by summing up the squared amplitudes of the eigenvector components at the corresponding vertex point $\left(a_{i j}\right)$ times a Gaussian function centered at the energy of the corresponding energy eigenvalue $\left(\varepsilon_{j}\right)$,

$$
\operatorname{LDOS}\left(r_{i}, \varepsilon\right)=\sum_{j}\left|a_{i}^{j}\right|^{2} \cdot \operatorname{Gauss}\left(\varepsilon-\varepsilon_{j}, \sigma\right) .
$$

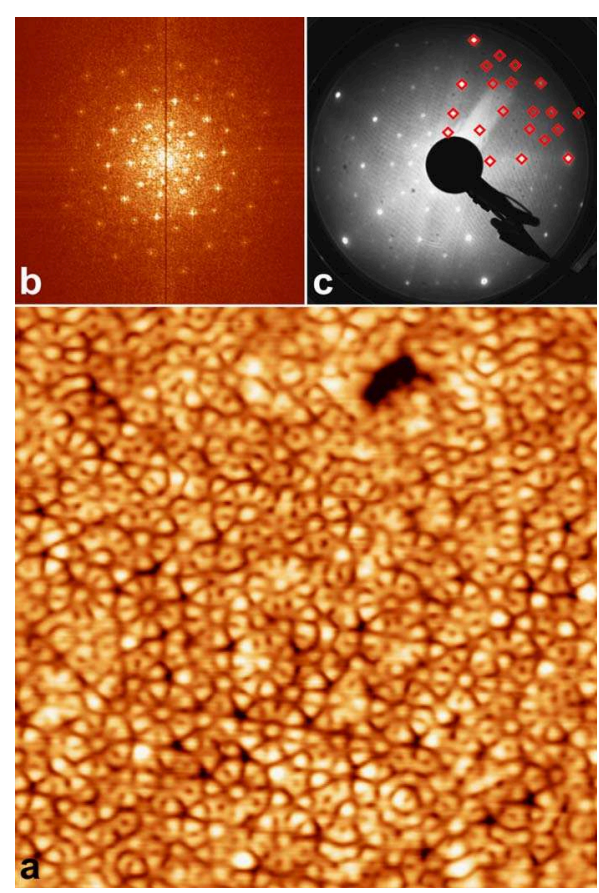

FIG. 1. (Color online) Topography of the fivefold AlPdMn surface. (a) High-resolution STM image of QC measured at $5 \mathrm{~K}$ with scan size $18 \times 18 \mathrm{~nm}^{2}, \Delta z: 0.3 \mathrm{~nm}, U_{T}=0.45 \mathrm{~V}$, and $I_{T}=0.1 \mathrm{nA}$, (b) FFT of STM image in (a) $\left(12.2 \times 12.2 \mathrm{~nm}^{-2}\right)$, and (c) LEED pattern of the freshly prepared QC recorded at $55 \mathrm{eV}$; the diamonds mark the position of the diffraction spots in one sector.

\section{RESULTS AND DISCUSSION}

Figure 1(a) shows a typical high-resolution STM image of a bare fivefold surface of the $\mathrm{Al}_{70} \mathrm{Pd}_{21} \mathrm{Mn}_{9} \mathrm{QC}$, revealing the characteristic features of this surface. The corresponding fast Fourier transformation (FFT) representation [Fig. 1(b)] of this STM image and the low-energy electron diffraction (LEED) [Fig. 1(c)] of the same surface taken before STM imaging reveal the fivefold symmetry, where the diffraction spots are arranged according to a LS Fibonacci sequence. These sequences are based on length of $S=0.24 \mathrm{~nm}$ and $L=S \tau=0.39 \mathrm{~nm}$, respectively, in real space, with $\tau$ being the golden mean equal to $1.61803 . .$. Both LEED and atomic resolution STM indicate the high quality of the prepared surface.

A clear identification of the main structural features as "dark stars" (cyan circle) and "white flowers" (dark blue circle $)^{29}$ is shown in Fig. 2(a). The correspondence of the atomic position of these features with the geometrical $M$ model $^{27}$ is highlighted in Fig. 2(b). The local structural motifs seen in the STM images can be found from the atomic models, where they are related to layers of Bergman and Mackay clusters above, below, and dissected by the terminating plane of the model. Therefore, the "white flowers" are composed by five Bergman polytopes surrounding a central dissected Mackay cluster; whereas the center of the "dark stars" is represented by a dissected Bergman polytope with a vacancy in the center. This assignment of the "dark stars" with the intersection of Bergman clusters is under debate and discussed extensively in a recent publication. ${ }^{30}$ 


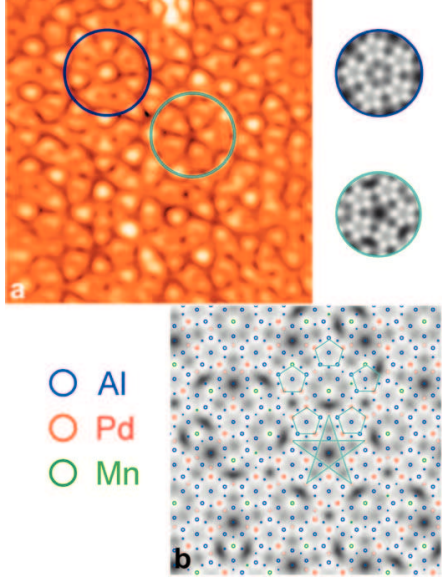

FIG. 2. (Color) High-resolution STM image of the fivefold AlPdMn surface. (a) High-resolution STM image of QC measured at $5 \mathrm{~K}$ with scan size $8 \times 8 \mathrm{~nm}^{2}, \Delta z: 0.2 \mathrm{~nm}, U_{T}=1 \mathrm{~V}$, and $I_{T}=84 \mathrm{pA}$. Blue and cyan circles identifying "white flower" and "dark star," respectively, in experiment and STM simulations. (b) Model structure where the elements $\mathrm{Al}, \mathrm{Pd}$, and $\mathrm{Mn}$ are represented by blue, red, and green circles, respectively. Three top layers according to the $M$ model forming the surface according to circle sizes.

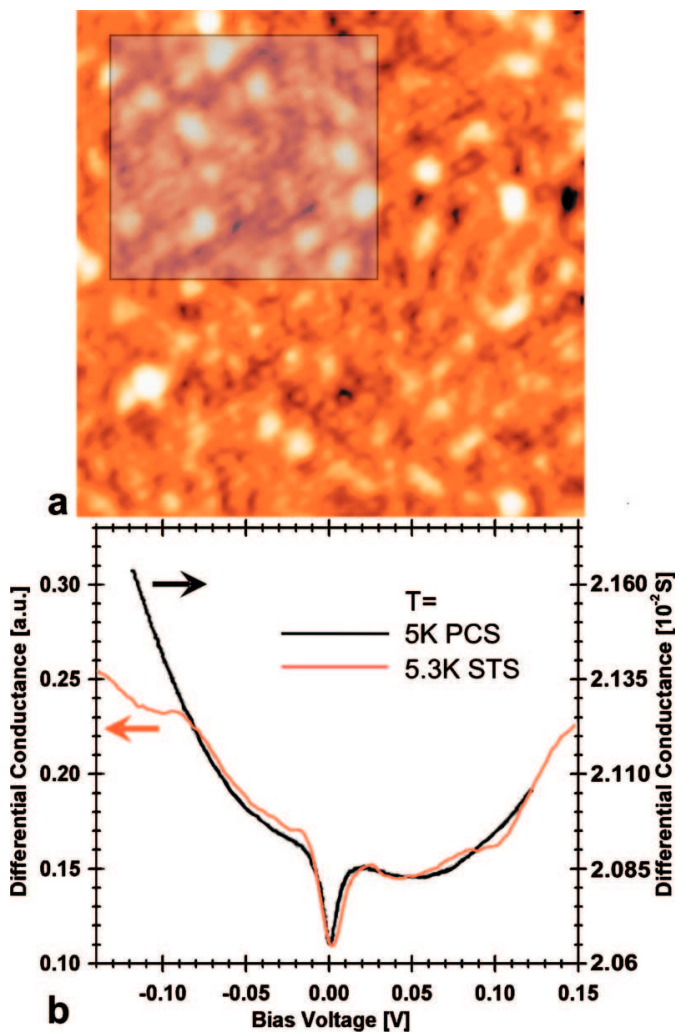

FIG. 3. (Color) Total density of states. (a) STM image of QC measured at $5 \mathrm{~K}$ with scan size $15 \times 15 \mathrm{~nm}^{2}, \Delta z: 0.2 \mathrm{~nm}, U_{T}$ $=0.4 \mathrm{~V}$, and $I_{T}=0.2 \mathrm{nA}$, with shaded inset of $8 \times 7 \mathrm{~nm}^{2}$ where a grid of 125 single STS spectra are measured, which is displayed in (b). (b) Differential conductance $d I / d V$ vs bias voltage, black line from Ref. 10 measured at $5 \mathrm{~K}$ by PCS belonging to the right vertical axis, red curve measured at $5 \mathrm{~K}$ by STS and averaged belonging to the left vertical axis, and energy range from $-0.14 \mathrm{~V}$ to $+0.15 \mathrm{~V}$.
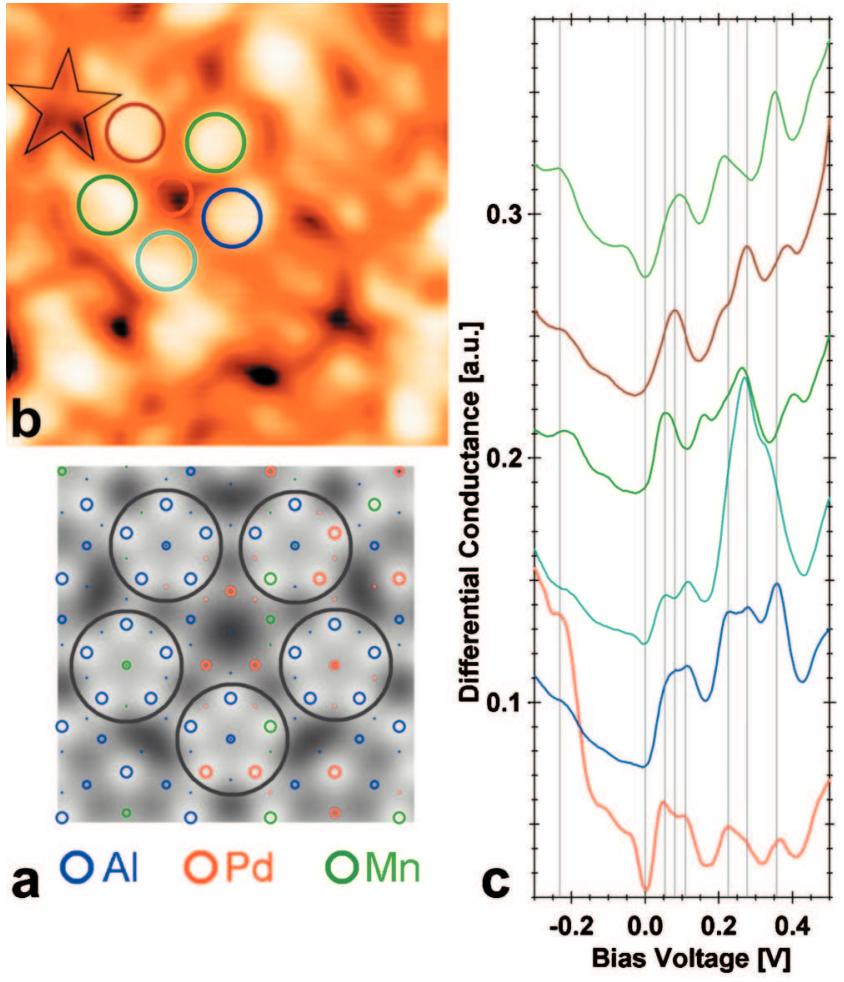

FIG. 4. (Color) Local density of states of the fivefold AlPdMn surface. (a) Model structure of a "dark star" demonstrating the chemical heterogeneity within the Bergman polytopes described with black circles. Al, Pd, and $\mathrm{Mn}$ are represented by blue, red, and green circles, respectively. Three top layers according to the $M$ model are mapped according to circle sizes. (b) STM image with star and circles identify the location of the STS spectra shown in (c); scan size: $4 \times 4 \mathrm{~nm}^{2}, \Delta z: 0.2 \mathrm{~nm}, U_{T}=0.5 \mathrm{~V}$, and $I_{T}$ $=0.2 \mathrm{nA}$. (c) Differential conductance $d I / d V$ vs bias voltage of LDOS colorized according to (b), and energy range from $-0.3 \mathrm{~V}$ to $+0.5 \mathrm{~V}$ offset by 0.05 arb. units in $y$ for better visibility.

The aperiodic structure of the QC has, as consequence, that every atom and of course also every surface atom is structurally inequivalent. The model structure of Fig. 2(b) further illustrates that additionally to the structural inequivalency there exists a pronounced chemical inequivalency, which can lift the structural similarity, e.g., of the five Bergman clusters at the periphery of the "dark star." Therefore, each atomic position is expected to have different valence electronic properties.

To probe these differences, we have performed LT-STS measurements using the lock-in technique on the clean fivefold and twofold surfaces of the AIPdMn QC with high spatial resolution. First we like to compare our STS results with previously published studies $^{10}$ performing point-contact spectroscopy (PCS). The acquired spectra of differential conductance vs the bias voltage represent, in the first approximation, the LDOS as a function of the electron energy, with zero bias corresponding to $E_{F}$ and the bias voltage corresponding to the electron energy in $\mathrm{eV}$. As the bias represents the potential of the sample with respect to the tip, positive bias corresponds to the unoccupied and negative bias to the occupied states. The shaded region in Fig. 3(a) marks an area 
of $8 \times 7 \mathrm{~nm}^{2}$ in an STM image of $15 \times 15 \mathrm{~nm}^{2}$ of the clean fivefold surface, where 125 single STS spectra were taken and averaged in order to obtain the red curve presented in Fig. 3(b). This averaging was performed to draw a comparison with the PCS measurements, where the surface was oxidized and the contact area is in the range of $1 \mu \mathrm{m}^{2}$. Figure 3(b) shows the comparison between our STS results at $5.3 \mathrm{~K}$ and the PCS curves from Escudero et al. ${ }^{10}$ at $5 \mathrm{~K}$ (black curve). The vanishing of fine structure with increasing temperature as well as the shape of our averaged STS curve on a clean surface are in very good qualitative agreement with the PCS curves from Escudero et al. ${ }^{10}$ on oxidized samples. Besides the onset of the large parabolic pseudogap over the whole spectra, a sharp and deep dip at $E_{F}$ is the most remarkable feature. This ZBA, which is a hallmark of the interactions between the electrons ${ }^{26}$ has a full width at half maximum of $18-20 \mathrm{meV}$ at $5 \mathrm{~K}$ and vanishes with increasing temperature. On each side of the ZBA, we observe little shoulders in comparison with the overall parabola of the large pseudogap. With increasing number and area over which the averaging is carried out, the fine structure still visible in the spectrum of Fig. 3(b) is vanishing and only the large parabolic pseudogap with the sharp dip at the Fermi energy remains. In agreement with newer theoretical calculations, ${ }^{9}$ there is no evidence for a spiky DOS in these averaged spectra.

Taking a closer look at the individual spectra (Fig. 4), the sharp and deep ZBA still can be identified but is not present in all the individual spectra. When comparing individual spectra with the averaged ones, the occurrence of a large number of sharp peaks and gaps not situated uniquely at $E_{F}$ any more is very apparent. The chemical heterogeneity at the surface is again schematically represented in Fig. 4(a) where five pentagons are marked with circles composing a "dark star." In this model representation, it is clearly seen that none of these Bergman polytopes has exactly the same chemical composition or environment as some consist only of $\mathrm{Al}$ and others also contain Pd and/or Mn. Even though the correct chemical composition of the terminating layers is still under discussion, ${ }^{30,31}$ the identification of the distinctive surface features allows the attribution of STS spectra to these specific sites. On a corresponding STM image shown in Fig. 4(b), a high-resolution STS grid was measured and circles are drawn to highlight the Bergman polytopes surrounding a "dark star" feature. The topographic resolution of this STM image is slightly lower than usual because a homogenously spaced grid of 900 individual STS spectra was simultaneously recorded. Figure 4(c) displays STS spectra averaged on the pentagonal areas around the "dark star" of Fig. 4(b) indicated by the circles (spectrum originates from the circle of same color).

In analogy with the averaged spectrum of Fig. 3(b), the distinct pseudogap at the Fermi level is also observed in the spectrum at the center of the star (red curve). The other spectra on the pentagons do not show this pronounced ZBA, at least not to the same extent. The narrow dip in the DOS shows at $\pm 50 \mathrm{meV}$ peaklike structures identified as the shoulders of the spectrum in Fig. 3(b). The shape of these accompanying peaks can become in some cases very intense and sharp exhibiting Fano-line shapes. ${ }^{32}$ It could be possible that these peaks are due to impurity tunneling or Kondo effect attributed to the Mn atoms. ${ }^{17,23}$ Such magnetic atoms in a nonmagnetic host have been investigated in the more simple $\mathrm{Co}$ on $\mathrm{Au}(111)$ system, where these narrow spectroscopic features close to $E_{F}$ also revealing a spatial extent of about $1 \mathrm{~nm}$ have been identified being Kondo resonances. ${ }^{33}$ A correlation between magnetic properties and electrical resistivity in QC has been demonstrated ${ }^{23,26}$ however, we like to stress that more investigations of this structures close to $E_{F}$ are needed to draw a firm conclusion. Turning our attention away from $E_{F}$, one can observe a multitude of peak and pseudogap structures, especially in the positive bias region.

As a function of topographic position, the spectral features change very rapidly, with a typical spatial extent of the order of $0.5 \mathrm{~nm}$. This situation can be qualitatively understood from the inequivanlence of every single surface site with regard to chemical composition and environment as discussed before. This means that although we identify five apparently equivalent regions around the dark star feature, being the pentagons of the dissected Bergmann polytopes, these sites can still be highly inequivalent despite their similarity in the STM topography. At present, the STM can only indicate structurally similar regions, but information on the chemical compositions of these regions can hardly be obtained. The variability of the STS spectra on such structurally similar regions might serve to elucidate the chemical state in future. However, we need to stress here that due to the complexity of the system, this chemical assignment could not yet be achieved.

From the data presented in Fig. 4, it becomes clear that the nature, i.e., the spatial confinement and spatial variation in the spectral features, can only be resolved with a highresolution spectrum grid measurement with a spatial resolution of the order to $0.1 \mathrm{~nm}$, as displayed in Fig. 5. Figure 5(a) represents a STM image of the fivefold surface with a field of view of $10 \times 10 \mathrm{~nm}^{2}$, where a high-resolution STS grid of 3000 individual spectra was simultaneously measured. The line cut representation of the differential conductance spectra shown in Fig. 5(b) on an extended energy range from $-0.6 \mathrm{~V}$ to $+0.6 \mathrm{~V}$ was extracted from the STM image of Fig. 5(a) along the blue line. The vertical axis in Fig. 5(b) represents sample bias (corresponding to energy, respectively), the horizontal axis the distance along the line cut highlighted in Fig. 5(a), and the normalized spectral intensity is plotted in color. As already mentioned above, the ZBA at $E_{F}$ can be seen in Fig. 5(b) as a fine dark line at zero bias; but it is evident that the localization of the states and pseudogaps is not only restricted to energies around $E_{F}$.

The spectral features observed in Fig. 5(b) do not show a "bandlike" behavior, which means that they do not shift into new energetic positions as a function of tip position. Rather the pseudogaps and peaks appear and disappear. Although a correlation is hardly visible from the spectral cut, it seems that there exist favored energies along this line cut. However, all energetic states appear globally with about the same frequency, which results in the smeared out total DOS spectrum shown in Fig. 3(b), where the single spectra were averaged over an extended surface region.

Concerning the fine structure in the STS spectra, another remarkable observation is the asymmetry between the occu- 


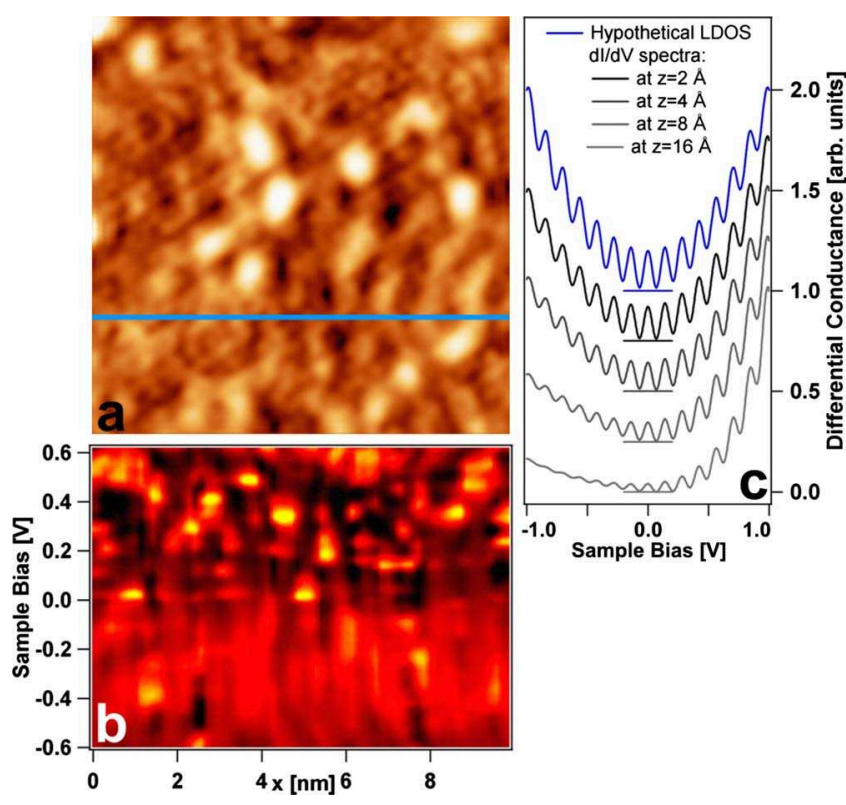

FIG. 5. (Color online) Line-cut representation of LDOS of the fivefold AlPdMn surface. (a) STM image where a grid of 3000 individual STS spectra is simultaneously measured with horizontal (blue) line representing the location of the map displayed in (b); scan size: $10 \times 10 \mathrm{~nm}^{2}, \Delta z: 0.2 \mathrm{~nm}, U_{T}=0.5 \mathrm{~V}$, and $I_{T}=0.2 \mathrm{nA}$. (b) Spatial-resolved energy map of the normalized differential conductance $d I / d V$ of LDOS along the blue line drawn in (a) with energy range from $-0.6 \mathrm{~V}$ to $0.6 \mathrm{~V}$; pseudogaps map is dark and states is bright. (c) Simulation of dependency of the differential conductance with tip-sample distance applying a hypothetical LDOS with a parabolic shape onto which a cosine oscillation emulates the observed spikiness.

pied and unoccupied states as the variation and peculiarity of the states above $E_{F}$ (positive bias) is very pronounced; whereas the spectrum below $E_{F}$ is rather smooth and featureless. This behavior is also present in Fig. 4(c) but not with the same distinctness. The asymmetry between occupied and unoccupied states does not only concern the variability in energy but also in the spatial extent of the spectral features. The spectral features, whether being pseudogaps or peaks, show up with a narrow energy confinement of roughly 30-50 $\mathrm{meV}$ and are localized to very small regions of the surface with a spatial extent in the order of $0.5 \mathrm{~nm}$, as seen in Fig. 5(b). The consequence of this behavior finds its expression in different STM imaging quality for negative and positive sample biases and the ability to assign local states to topographic STM information.

The origin of the distinct difference of the spectroscopic signatures between the occupied and unoccupied states can be related to a fundamental property of the tunneling spectroscopy, as we show here after. At the basis of our consideration, we construct a hypothetical LDOS with a parabolic shape onto which a cosine oscillation emulates the spikiness we observe [see solid blue line Fig. 5(c)]. Obviously this LDOS is symmetric with respect to zero bias (the Fermi energy). In Fig. 5(c), we now compare the simulated differential conductance spectra at various tip-sample distances with the genuine LDOS which enters the simulation. The

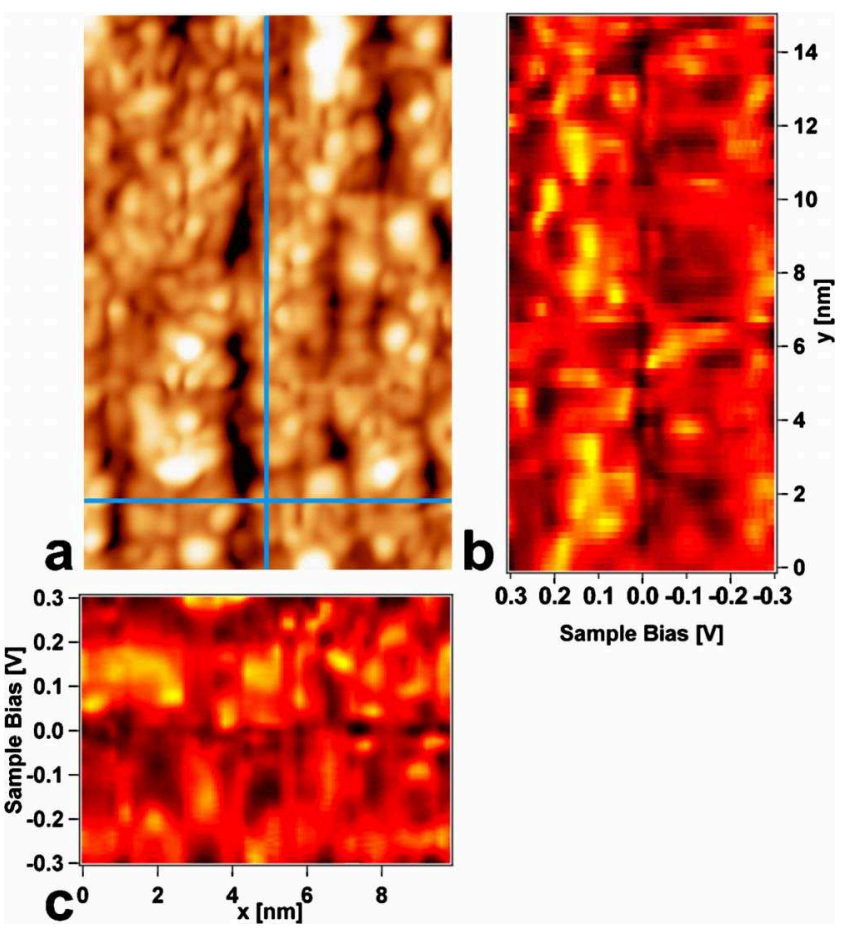

FIG. 6. (Color online) Line-cut representation of LDOS of the twofold AlPdMn surface. (a) STM image where a grid of 3000 individual STS spectra is simultaneously measured with the blue line representing the location of the map displayed in (b); scan size: $10 \times 15 \mathrm{~nm}^{2}, \Delta z: 0.2 \mathrm{~nm}, U_{T}=0.3 \mathrm{~V}$, and $I_{T}=0.1 \mathrm{nA}$. (b) Spatialresolved energy map of the normalized differential conductance $d I / d V$ of LDOS along the vertical blue line drawn in (a) with energy range from $-0.3 \mathrm{~V}$ to $0.3 \mathrm{~V}$; pseudogaps map is dark and states are bright. (c) Analog to (b) along the horizontal blue line drawn in (a).

basis for the simulation is the well-known semiclassical Tersoff-Hamann ${ }^{34}$ expression for the tunneling current density,

$$
\begin{aligned}
J(z, V) \cong & \frac{2 \pi \cdot e}{\hbar}\left(\frac{\hbar^{2}}{2 m}\right)^{2} \int_{-\infty}^{\infty} T(z, V, E)[f(E-e V)-f(E)] \\
& \times \rho_{s}(E) \rho_{t}(E-e V) d E,
\end{aligned}
$$

where $T(z, V, E)$ is the tunneling transmission probability in the Wentzel, Kramers, and Brillouin approximation, and $\rho_{s}(E)$ and $\rho_{t}(E)$ are the surface and tip DOS, respectively.

By increasing the tip-sample distance, a slight enhancement of the oscillation at very positive sample biases is observed. On the other hand and even more important is the pronounced damping in the negative potential region. This is because of the decay of the transmission probability which becomes the decisive parameter in the tunneling process between tip and sample. To probe the occupied states of the sample, $E_{F}$ of the sample is shifted negative with respect to the tip and the tunneling into the unoccupied states of the tip is rigorously reduced by the decreasing transmission probability. This effect becomes even more pronounced with larger tip-sample separations and explains in this way the obtained experimental results. 

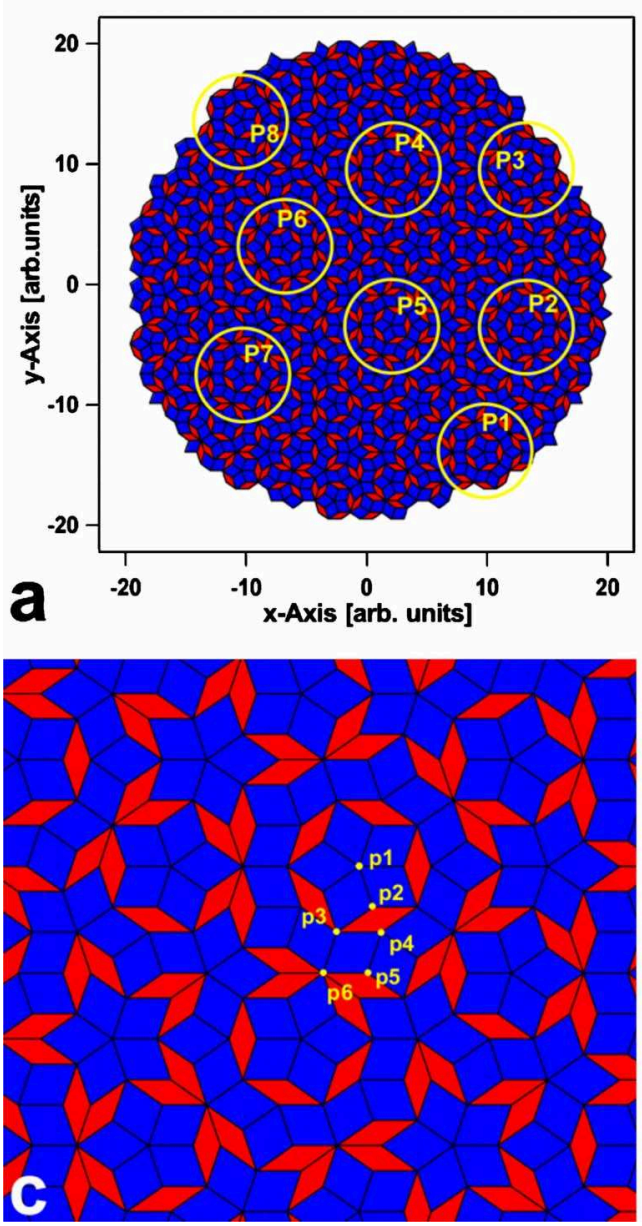

To support the statements from above and to further explore the AlPdMn system, we performed STS investigations on the twofold AlPdMn surface, which are presented in Fig. 6 analog to Fig. 5. Figure 6(a) shows a $10 \times 15 \mathrm{~nm}^{2}$ STM image of the twofold surface, where again a high-resolution STS grid of 3000 individual spectra was simultaneously acquired. The line cut representation of the differential conductance spectra shown in Figs. 6(b) and 6(c) on an energy range from $-0.3 \mathrm{~V}$ to $+0.3 \mathrm{~V}$ were extracted from the STM image of Fig. 6(a) along the vertical and horizontal blue lines, respectively, which represent the two high-symmetry directions.

As discussed above, the asymmetry between occupied and unoccupied states is also visible on the twofold AlPdMn surface, just to a lesser extent because of the narrower energy window. Analog to Fig. 5(b), we observe a high variety of localized states and pseudogaps and also the ZBA at $E_{F}$ is present on this surface as can be seen as a fine dark line at zero bias. Even though we do not observe a "bandlike" behavior of the spectral features, it seems that there exist favored energies along these line cuts as discussed in Fig. 5(b). Also the energy confinement of some states and pseudogaps of roughly $30-50 \mathrm{meV}$ and the spatial localization in the order of $0.5 \mathrm{~nm}$ are preserved. This analogy between the fivefold and twofold surfaces, which stand perpendicular to
FIG. 7. (Color online) Tightbinding calculations of LDOS. (a) Finite patch of the twodimensional (2D) Penrose lattice with eight locally similar regions highlighted by yellow circles and labeled from P1-P8. (b) TB calculation of the LDOS at the central vertex points (light blue marker in the inset) of the pattern $\mathrm{P} 1-\mathrm{P} 8$ from (a). (c) Detail of the 2D Penrose lattice with six neighboring vertex points p1-p6 labeled. (d) The TB LDOS corresponding to the vertex points $\mathrm{p} 1-\mathrm{p} 6$ from (c). each other, emphasizes the validity of the observations for the AlPdMn system.

The appearance of peaks in the LDOS of QC can be qualitatively predicted and described by tight-binding calculations as shown in Fig. 7. Figure 7 displays the findings of the TB calculations on a finite patch of the Penrose tiling, where the LDOS of structurally similar vertexes are compared [P1 to P8 in Fig. 7(a)]. According to Conway's theorem, ${ }^{35}$ a selected pattern of radius $r$ can be found within a quasiperiodic lattice at a mean distance of $2 r$. This is reflected in the distribution of the "rosettelike" pattern considered here [see inset in Fig. 7(b)]. These patterns however show different surroundings if a region larger than $r$ is considered. Figure 7(b) displays the LDOS calculated as described in Sec. II in the center of the "rosette"-pattern [light blue marker in the inset of Fig. 7(b)]. The spectra displayed in Fig. 7(b) have been offset for better visibility. It can be observed that the LDOS in the center of the considered pattern is rather similar, although not identical, as all the points P1-P8 are still inequivalent although with similar surroundings.

Additionally a different set of vertex points [p1 to p6 in Fig. $7(\mathrm{c})]$ in rather close proximity to each other has been considered with the corresponding LDOS spectra displayed in Fig. 7(d), again the spectra are offset for better visibility. 
The local spikiness of the spectra is readily observed, which can be explained by the larger structural inequivalency/ aperiodicity as compared to the vertex points considered in Fig. 7(a). These qualitative findings agree well with our experimental findings of a locally extremely variable electronic structure. However, a second feature of the simulation-the self-similarity-is not easily found in the experiment. Here we need to be aware of the chemical heterogeneity of the material, and in this context, not only the topmost atomic layer but also deeper layers will contribute to the LDOS. This chemical heterogeneity will obviously preserve the spatial variability and will otherwise lift the structural selfsimilarity in many cases. This means that local atomic arrangements of the same geometrical structure [which may look similar in topographic STM, compare Fig. 4(b)] will be different with regard to the chemical composition.

\section{CONCLUSIONS}

In contrast to other experimental work on tunneling spectroscopy of QC, we investigated the LDOS of the clean and well-characterized fivefold and twofold surfaces of $i$-AlPdMn QC using low temperature $(5.3 \mathrm{~K})$ STS with subnanometer resolution. Averaging STS spectra over surfaces larger than $6 \times 6 \mathrm{~nm}^{2}$ results in differential conductance spectra, which are in good agreement with previously published spectra obtained from PCS measurements. The characteristic features of these averaged spectra are a large parabolic pseudogap with a narrow dip of 20-30 meV width at zero bias identified as a zero-bias anomaly. In contrast, the individual local spectra representing the LDOS show a large number of energetically as well as spatially well-localized features, resembling a spiky DOS instead of the rather smooth averaged spectra.

We could demonstrate that this localization of states and pseudogaps occurs on the fivefold as well as on the twofold AlPdMn surface and applies also for the ZBA at $E_{F}$ with a typical extent in the order of $0.5 \mathrm{~nm}$. The distinct asymmetry between the occupied and the unoccupied LDOSs could be attributed to the tunneling transmission probability. Although much more effort has to be devoted to the subject of the appearance of sharp spatially localized electronic states, the qualitative reproduction using a tight-binding model could be performed, where geometrically similar sites on the QC surface produced strongly different LDOS taking into account the dissimilar chemical composition of these sites.

The spectra averaged over a larger surface area could be considered to approach the total DOS, which results in an appearance of the averaged spectrum consistent also with newer theoretical calculations. On the other hand, the single spectra of the local DOS reveal a spiky DOS as predicted in earlier theoretical calculations.

The ternary quasicrystalline AlPdMn system reveals a very complex nature with many unknown parameters. To approach the problem of attributing spectral features to the topography, so-called approximant phases of the QC offer promising perspectives, as they have a finite unit cell.

\section{ACKNOWLEDGMENTS}

This work was partially supported by the European Community through the SMART Quasicrystals Project No. G5RD-CT-2001-00584 and the Swiss National Foundation (Contract No. SNF 200021-112333/1), whose financial support is greatly appreciated. *roland.widmer@empa.ch

${ }^{1}$ D. Shechtman, I. Blech, D. Gratias, and J. W. Cahn, Phys. Rev. Lett. 53, 1951 (1984).

${ }^{2}$ Quasicrystals: An Introduction to Structure, Physical Properties and Applications, Springer Series in Materials Science Vol. 55, edited by J.-B. Suck, M. Schreiber, and P. Häusler (Springer, Berlin, 2002).

${ }^{3}$ J. Delahaye and C. Berger, Phys. Rev. B 64, 094203 (2001); J. Delahaye, C. Berger, and G. Fourcaudot, J. Phys.: Condens. Matter 15, 8753 (2003).

${ }^{4}$ M. de Boissieu, M. Boudard, H. Moudden, M. Quilichini, R. Bellissent, B. Hennion, R. Currat, A. Goldman, C. Janot, J. NonCryst. Solids 153-154, 552 (1993).

${ }^{5}$ Y. Akahama, Y. Mori, M. Kobayashi, H. Kawamura, K. Kimura, and S. Takeuchi, J. Phys. Soc. Jpn. 58, 2231 (1989).

${ }^{6}$ S. Yamamoto and T. Fujiwara, Phys. Rev. B 51, 8841 (1995); S. Roche and D. Mayou, Phys. Rev. Lett. 79, 2518 (1997).

${ }^{7}$ G. Trambly de Laissardiere, J. P. Julien, and D. Mayou, Phys. Rev. Lett. 97, 026601 (2006).

${ }^{8}$ T. Fujiwara and T. Yokokawa, Phys. Rev. Lett. 66, 333 (1991).

${ }^{9}$ E. S. Zijlstra and T. Janssen, Mater. Sci. Eng., A 294-296, 886 (2000) and references therein.

${ }^{10}$ R. Escudero, J. C. Lasjaunias, Y. Calvayrac, and M. Boudard, J.
Phys.: Condens. Matter 11, 383 (1999).

${ }^{11}$ T. Klein, O. G. Symko, D. N. Davydov, and A. G. M. Jansen, Phys. Rev. Lett. 74, 3656 (1995).

${ }^{12}$ L. Guohong, H. Haifeng, W. Yunping, L. Li, L. Shanlin, J. Xiunian, and Z. Dianlin, Phys. Rev. Lett. 82, 1229 (1999).

${ }^{13}$ J. Delahaye, T. Schaub, C. Berger, and Y. Calvayrac, Phys. Rev. B 67, 214201 (2003) and references therein.

${ }^{14}$ T. Schaub, J. Delahaye, C. Ginoux, C. Berger, C. Fourcaudot, F. Giroud, T. Grenet, and A. G. M. Jansen, J. Non-Cryst. Solids 250-252, 874 (1999).

${ }^{15}$ D. N. Davydov, D. Mayou, C. Berger, C. Ginoux, and A. G. M. Jansen, Mater. Sci. Eng., A 226-228, 972 (1997).

${ }^{16}$ D. N. Davydov, D. Mayou, C. Berger, C. Gignoux, A. Neumann, A. G. M. Jansen, and P. Wyder, Phys. Rev. Lett. 77, 3173 (1996).

${ }^{17}$ G. N. Banerjee, S. Banerjee, and R. Goswami, J. Non-Cryst. Solids 334-335, 388 (2004).

${ }^{18}$ J. Yuhara, J. Klikovits, M. Schmid, P. Varga, Y. Yokoyama, T. Shishido, and K. Soda, Phys. Rev. B 70, 024203 (2004).

${ }^{19}$ J. Dolinsek, M. Klanjsek, T. Apih, A. Smontara, J. C. Lasjaunias, J. M. Dubois, and S. J. Poon, Phys. Rev. B 62, 8862 (2000); Z. M. Stadnik, D. Purdie, Y. Baer, and T. A. Lograsso, ibid. 64 214202 (2001) 
${ }^{20}$ R. Widmer, O. Gröning, P. Ruffieux, and P. Gröning, Philos. Mag. 86, 781 (2006).

${ }^{21}$ Z. M. Stadnik, D. Purdie, M. Garnier, Y. Baer, A. P. Tsai, A. Inoue, K. Edagawa, S. Takeuchi, and K. H. J. Buschow, Phys. Rev. B 55, 10938 (1997) and references therein.

${ }^{22}$ T. Fujiwara, in Physical Properties of Quasicrystals, Springer Series in Solid State Sciences Vol. 166, edited by Z. M. Stadnik (Springer, Berlin, 1999), pp. 169-207; X. Fu, X. Zhanga, and Z. Hou, J. Non-Cryst. Solids 354, 1740 (2008).

${ }^{23}$ G. Trambly de Laissardière, D. Nguyen-Manh, and D. Mayou, Prog. Mater. Sci. 50, 679 (2005).

${ }^{24}$ M. Krajci and J. Hafner, Phys. Rev. B 71, 054202 (2005) and references therein.

${ }^{25}$ J. J. Prejean, C. Berger, A. Sulpice, and Y. Calvayrac, Phys. Rev. B 65, 140203(R) (2002).

${ }^{26}$ B. L. Altshuler and A. G. Aronov, in Electron-Electron Interactions in Disordered Systems, edited by B. A. L. Efros and M. Pollak (Elsevier, Amsterdam, 1985).

${ }^{27}$ G. Kasner, Z. Papadopolos, P. Kramer, and D. E. Bürgler, Phys.
Rev. B 60, 3899 (1999).

${ }^{28}$ O. Gröning, R. Widmer, P. Ruffieux, and P. Gröning, Philos. Mag. 86, 773 (2006).

${ }^{29}$ Z. Papadopolos, G. Kasner, J. Ledieu, E. J. Cox, N. V. Richardson, Q. Chen, R. D. Diehl, T. A. Lograsso, A. R. Ross, and R. McGrath, Phys. Rev. B 66, 184207 (2002).

${ }^{30}$ Z. Papadopolos, R. Widmer, and O. Gröning, Philos. Mag. 88, 2083 (2008).

${ }^{31}$ B. Unal, C. J. Jenks, and P. A. Thiel, Phys. Rev. B 77, 195419 (2008).

${ }^{32}$ U. Fano, Phys. Rev. 124, 1866 (1961).

${ }^{33}$ V. Madhavan, W. Chen, T. Jamneala, M. F. Crommie, and N. S. Wingreen, Science 280, 567 (1998).

${ }^{34}$ J. Tersoff and D. R. Hamann, Phys. Rev. Lett. 50, 1998 (1983); A. Selloni, P. Carnevali, E. Tosatti, and C. D. Chen, Phys. Rev. B 31, 2602 (1985); V. A. Ukraintsev, ibid. 53, 11176 (1996).

${ }^{35}$ M. Gardner, Sci. Am. 236, 110 (1977). 